\title{
Physics of Switching and Memory Effects in Chalcogenides
}

\author{
K. D. Tsendin \\ Ioffe Physical_Technical Institute, Russian Academy of Sciences, ul. Polytekhnicheskaya 26, St.Petersburg, 194021, Russia \\ *Corresponding Author: tsendin@mail.ioffe.ru
}

Copyright $@ 2014$ Horizon Research Publishing All rights reserved.

\begin{abstract}
A review of the main experimental features of switching and memory effects in chalcogenide glassy semiconductors (CGS), review and analyze of the models of these phenomena was done. An electronic-thermal model of the switching effect taking into account multiphonon tunnel ionization of negative- $U$ centers and heating is presented. The model fits experimental current-voltage characteristics of a GeSbTe films both in ohmic and exponential regions. Also the model is in good agreement with experimental dependences of threshold voltage and threshold current on temperature and thickness. This indicates that multiphonon tunnel ionization of negative- $U$ centers and heating is the most probable mechanism of the switching effect.
\end{abstract}

Keywords Chalcogenides, Switching and Memory Effects

\section{Introduction}

Fifty years, since the early 1960s [1-3] physics of switching and memory effects in chalcogenides have been widely discussed in many papers but the microscopic mechanism of these phenomena is unknown up to now. In the present paper it will be enumerated a lot of models which were put forward to explain the switching and memory effects in chalcogenides. It is obvious that to judge the validity of any model it should be compared in details with experimental features but one has to convince that such comparison is absent for the most number of models and uncertainty of the microscopic mechanism of phenomena is due to this absence.

In our recent papers we have carried out the comprehensive comparison of switching effect experimental features with electronic-thermal model taking into account a multiphonon tunneling ionization of negative- $U$ centers and Joule heating. In the present paper we would like to summarize these results. In the beginning the definition of switching and memory effects in chalcogenides will be done. Then the model preferences which are influenced us to the electronic-thermal model choice will be considered. The most important conclusion of the present paper is the following: numerous experimental features of switching effect may be explained by the electronic-thermal model which takes into account the multiphonon tunneling ionization of negative- $U$ centers and Joule heating. In the end of the paper a short review of today application progress will be done.

\section{Switching and Memory Effects}

Let us consider thin chalcogenide glassy semiconductor (CGS) film with thickness $\mathrm{L}$ which is under voltage $\mathrm{V}$. When the voltage is equal to threshold voltage $V_{t h}$ (Fig. 1a,b), then after delay time $t_{d}$ very fast transition (switching effect) takes place during the time $t_{S W}$, which is sufficiently less than $t_{d}\left(t_{S W} \ll t_{d}\right)$ from high resistance OFF state $\left(I_{t h}\right)$ to low resistance ON state $\left(I_{o n}\right)$. In the ON state there is minimal voltage - holding voltage $V_{h}$. If $\mathrm{V}<V_{h}$ then after restore time $\left(t_{r}\right.$, Fig. 1a) the OFF state restores. For this reason another name of switching effect is reversible breakdown. There are chalcogenide compounds, which after recording time $\left(\mathrm{t}_{\mathrm{set}}\right)$ memorize the ON state (Fig 1b, memory effect). Then one can see that switching effect is the first stage of the memory effect. Experimental patterns of switching and memory effects are presented in [4-7]. 


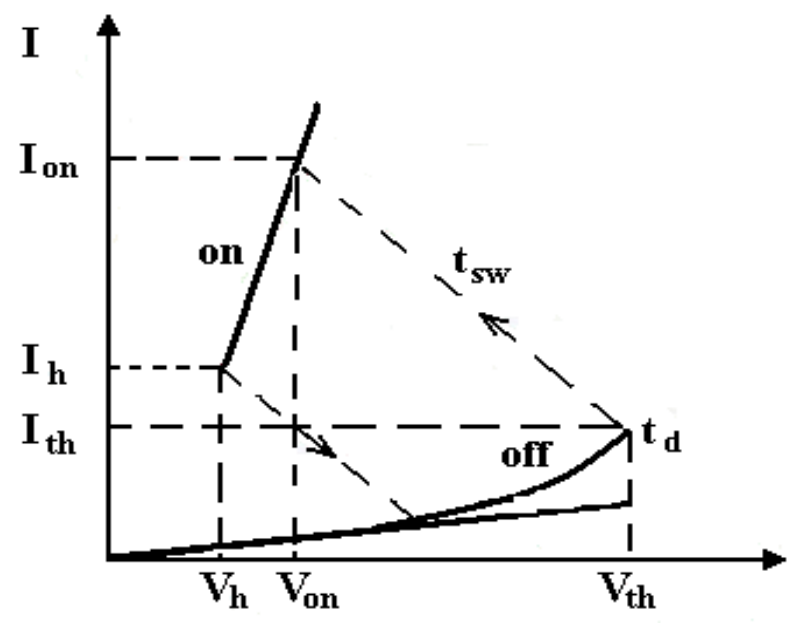

a

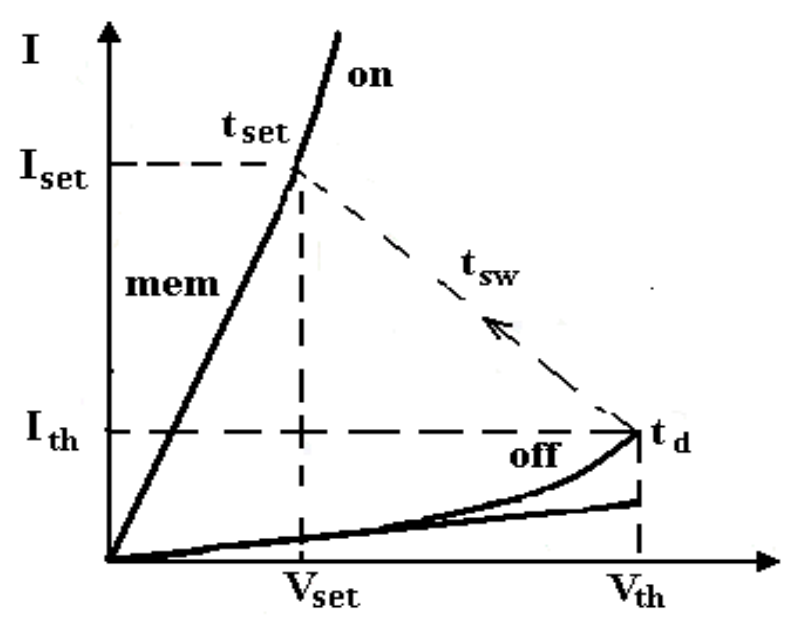

b

Figure 1. Current voltage characteristics (CVC) of switching (a) and memory (b) effects

\section{Theories and Models}

\subsection{Weak Fields Properties}

Let us consider the current voltage characteristics of Fig. 1 in a weak electric field (ohmic law).

It is very well known that energy activation of ohmic law conductivity $\Delta \mathrm{E}$

$$
\Delta E=\left(\frac{\varepsilon_{1}+\varepsilon_{2}}{2}\right)
$$

is governed in CGS by negative- $U$ centers - specific intrinsic defects of chalcogenide glassy semiconductors. Here $\varepsilon_{1}, \varepsilon_{2}$ are the first and second ionization energies of negative- $U$ centers. The strongest evidence of this is the independency of $\Delta \mathrm{E}$ on impurities and approximate coincidence of this value obtained in different laboratories, because negative- $U$ centers pin a Fermi level.

\subsection{Strong Field Properties}

To explain the strong increase in the conductance at the threshold point and the approximately exponential dependence of the conductance on a strong electric field (CVC nonlinearity, see OFF state conductivity deviation from linear ohmic law in Fig 1a,b), it was proposed that the CGS conductivity can be described by the phenomenological expression $[8,9]$

$$
I=I_{0} \cdot \exp \left(-\frac{\Delta E}{k T}+\frac{F}{F_{0}}\right)=I_{0} \cdot \exp \left(-\frac{\Delta E^{*}(F)}{k T}\right)
$$

In this expression, the first term in the exponent describes the activated-type conduction in a weak electric field; the second term defines the field dependence of the conductance in a strong electric field. There are several processes which could be described by the (2) rule. Among them are:

- Poole-Frenkel effect for the nearly situated local centers

- Non-linear hopping conduction along states near Fermi level

- Space charge limited current

- Decreasing of percolation energy

- Conductivity along crystalline species embedded into amorphous environment

The detailed review of current voltage nonlinearity may be found in [7]

\subsection{Electronic Models}

There are a lot of pure electronic models of switching effect, namely:

- One of the first electronic models of the switching effect was the double injection model proposed in 1969 independently by Mott [10] and Henisch [11].

- A similar switching model was described in a recent paper by Fritzsche [12]. In this model, the start of switching is associated with a Zener breakdown, and the high carrier concentration in the on state is provided by Fowler-Nordheim emission from contacts near which the field is the highest.

- It is noteworthy that Kolomiets and Lebedev as early as in 1963 stated the assumption about the relation of the negative differential resistance in CGSs to carrier injection [2].

- In 1970, Sandomirskii et al. proposed a model of the electronic phase transition [13]. They assumed that an increase in the free electron density results in a shift and disappearance of local level or the narrow band

- Ovshinsky was the first to suggest that memory effect is connected with crystallization [3].

- A switching mechanism based on impact ionization in two-level structures, even before the advent of the negative-U centers concept, was proposed in the study by Zabrodskii et al. [14]. They noticed that a 
switching effect associated with impact ionization is observed in crystalline semiconductors, in particular, in heavily doped compensated germanium at low temperatures. Therefore, the authors [14] assumed that a similar mechanism can underlie the switching effect in CGSs.

- Ryvkin [15] suggested to consider an amorphous semiconductor as a material in which potential fluctuations comparable to the band gap arise due to disorder in atomic positions. Therefore, the material consists of alternating $p$ and $n$ type regions and exhibits an $S$-shaped $I-V$ characteristics.

- In the paper by Adler et al. [16], two switching effect models based on impact ionization were suggested. In the first model, the possibility of the $S$ shape in a structure with two levels was qualitatively shown.

The second model comprehensively describes switching in a structure with negative- $U$ centers.

- The model of the switching effect, proposed by Ielmini and Zhang $[17,18]$, is based on thermally activated hopping conduction between localized states. Assuming that the density of localized states in CGS is high, $\sim 10^{18}-10^{19} \mathrm{~cm}^{-3}$, the average distance between such states which manifest themselves as carrier trapping centers is less than 10 $\mathrm{nm}$. In this case, charge transfer can occur via carrier tunneling between centers; at room temperature, thermally activated tunneling is most probable. In this case, tunneling occurs from a certain excited energy level, rather than from the ground level.

- According to the models of switching and memory effects, suggested in $[19,20]$, the crystallization energy barrier decreases in an electric field, therefore, a cylindrical crystal nucleus is rather rapidly formed in a high electric field.

All abovementioned theories and models are very interesting but one has no information on comprehensive comparison of these theories with experimental data presented on Figures 2-9.

\subsection{Electronic-Thermal Models}

Several electronic-thermal mechanisms for the switching effect were proposed.

- Male and Warren [21] were first to use expression (2) in the thermal theory of the switching effect. Later on, models in which the material conductivity is described by such an expression as (2) began to be referred to as electronic-thermal models of the switching effect, since, along with the thermal effects, they considered electronic processes leading to the explicit dependence of conductivity on the applied electric field.

- Kolomiets et al. [22] studied the dependence of the conductivity of $\mathrm{Si}_{12} \mathrm{Te}_{48} \mathrm{As}_{30} \mathrm{Ge}_{10}$ (STAG) thin films with injecting electrodes on the thickness and applied voltage. Based on these studies, it was shown that the space-charge-limited current can be a possible conductivity mechanism. In [22] it was theoretically shown that there is a critical film thickness at which threshold and holding voltages become equal, and the $S$-shaped behavior of $I-V$ characteristics is not observed in thinner films at all.

- The model, based on Auger recombination at negative- $U$ centers, is described in the study by Savransky [23].

One can see that there are more than ten models. To judge the validity of any model it should be compared in details with known experimental results. Such comparison is absent for the most number of models.

In our last decade papers [24-29] we have carried out the comprehensive comparison of switching effects experimental features with electronic-thermal model taking into account a multiphonon tunneling ionization (MTI) of negative-U centers and Joule heating. Now we would like to summarize these results.

\section{Electronic-Thermal Model Which Takes into Account a Multiphonon Tunneling Ionization of Negative-U Centers and Joule Heating}

\subsection{Theory}

Let us emphasize and consider arguments for electronic-thermal model which takes into account a multiphonon tunneling ionization (MTI) of negative-U centers and Joule heating. It is very well known that in a weak electric field energy activation of CGS conductivity $\Delta \mathrm{E}$ is governed by negative- $\mathrm{U}$ centers - specific intrinsic defects of chalcogenide glassy semiconductors (1). If one use the volume concentration of negative-U centers $10^{18} \mathrm{~cm}^{-3}$ then in a cell volume $3 \times 20 \times 20 \mathrm{~nm}^{3} \sim 10^{3} \mathrm{~nm}^{3}$ we obtain only one (!) negative-U center. So for pinning Fermi level one has to suggest that concentration is larger than $10^{18} \mathrm{~cm}^{-3}$. It may be, for example, due to generation of negative- $U$ centers near the cells boundary.

Shortly after discovery of the switching effect, many researchers assumed that the switching effect is a reversible thermal breakdown of a material $[30,31]$. It should be noted that, in contrast to thermal breakdown in insulators, the CGS film, after voltage removal, returned to the initial OFF state again (Fig.1a), therefore, the thermal breakdown in CGS was referred to as a reversible breakdown.

It is known that for S-shape current voltage characteristics within the framework of thermal breakdown heating up to the threshold temperature $T_{\text {th }}$ is necessary

$$
\mathrm{T}_{\text {th }}=\mathrm{T}_{0}+\Delta \mathrm{T}=\mathrm{T}_{0}+\mathrm{kT}_{0}{ }^{2} / \Delta \mathrm{E}
$$

Where $\Delta \mathrm{T}$ is rather small $(\sim 20 \mathrm{~K})$ for room temperature $\mathrm{T}_{0}=$ $300 \mathrm{~K}$ and for energy activation of conductivity $\Delta \mathrm{E}=0.4 \mathrm{eV}$. Value $\Delta \mathrm{T}$ is proportional to $1 /(\Delta \mathrm{E})$, then for 
electronic-thermal model when effective energy activation of conductivity $\Delta \mathrm{E}^{*}$ decreases due to electric field (2) $\Delta \mathrm{T}$ may be equal to $\sim 30-70 \mathrm{~K}$. These values are rather small and it is very difficult to prevent such heating.

The second argument for electronic-thermal switching model is sharp decrease of $V_{\text {th }}$ dependencies on $\mathrm{T}_{0}$ and $\mathrm{L}$. This decrease have been observed experimentally and was firstly emphasized and theoretically explained in paper [21].

Let us consider a CGS film to which an electric field is applied. In a strong field, the field induced ionization negative-U center will occur; therefore, in our opinion, the nonlinear conductivity of CGS in high electric fields can be associated with field induced ionization and carrier capture. For clarity, in this section, we will describe ionization and capture in terms of holes; similar expressions can be written for the case of ionization and capture of electrons. In this analysis, we will use results obtained in Ioffe institute by the Perel' team [32]. Let us first consider negative - U centers in the $D^{+}$and $D^{0}$ states which can donate one hole and transform to $D^{0}$ and $D^{-}$states respectively:

$$
\begin{aligned}
& D^{+}-p \leftrightarrow D^{0} \\
& D^{0}-p \leftrightarrow D^{-}
\end{aligned}
$$

Let $\left[D^{+}\right],\left[D^{0}\right]$, and $\left[D^{-}\right]$be the concentrations of centers in the $D^{+}, D^{0}$, and $D^{-}$states, respectively; then the active mass equations can be written as

$$
\begin{gathered}
\frac{\left[D^{0}\right] p}{\left[D^{+}\right]}=N_{V} \cdot \exp \left(-\frac{\varepsilon_{1}}{K T}+\omega_{1}\right) \\
\frac{\left[D^{-}\right] p}{\left[D^{0}\right]}=N_{V} \cdot \exp \left(-\frac{\varepsilon_{2}}{K T}+\omega_{2}\right) \\
{[D]=\left[D^{+}\right]+\left[D^{0}\right]+\left[D^{-}\right]} \\
{\left[D^{-}\right]=\left[D^{+}\right]+p .} \\
p=N_{V} \cdot \exp \left(-\frac{\varepsilon_{1}+\varepsilon_{2}}{2 K T}+\frac{\omega_{1}+\omega_{2}}{2}\right) . \\
j=\operatorname{ep}(T) \mu F=\sigma F
\end{gathered}
$$

The probability of the MTI of the centers $\omega_{1,2}$ has been calculated in [32]. Here, $p$ is the concentration of holes, $\mathrm{N}_{\mathrm{v}}$ is the effective electron concentration at the valence-band edge, $\varepsilon 1$ and $\varepsilon 2$ are the ground energy levels of the $D^{+}$and $D^{0}$ centers, respectively, $\omega_{1,2}$ give the numerically calculated increase of the ionization probability in the electric field $F$. If we assume that the concentration of holes is small compared to the concentration of the centers then we will obtain the concentration of holes (4). For simplicity, we suppose that the sample is spatially homogeneous. Also, we assume that the temperature of different points of the film is equal instead of solving the heat-conduction equation. Under these assumptions the steady-state temperature of the film can be found from the equality between the Joule heating and heat dissipation.

$$
F^{2} \sigma L=\lambda\left(T-T_{0}\right)
$$

Here, $L$ is the CGS film thickness, $T_{0}$ is the ambient temperature and $\lambda$ is the heat-exchange coefficient between the film and electrodes. This equation can be numerically solved with respect to the film temperature $T$ for each value of the electric field $F$. At certain values of the field, the equation has three solutions, which corresponds to the $S$-shaped $I-V$ characteristic (switching effect). Knowing the temperature, we calculate the hole concentration and substitute the obtained value into the expression for conductivity. Thus, we obtain the dependence of the CGS film conductivity on the applied electric field.

\subsection{Comparison with Experimental Data}

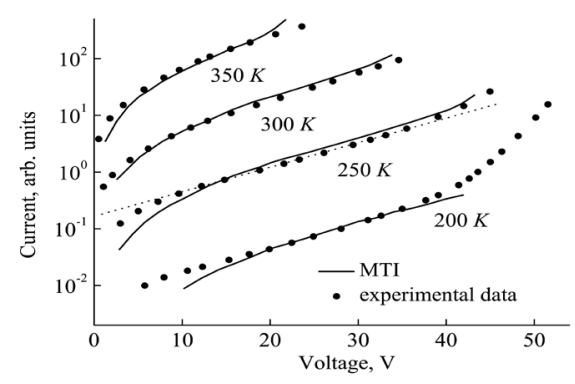

Figure 2. Current-voltage characteristics of a $\mathrm{Si}_{12} \mathrm{Te}_{48} \mathrm{As}_{30} \mathrm{Ge}_{10}$ (STAG) film $1.2 \mu \mathrm{m}$ thick at various temperatures. Solid lines are theoretical values for electronic-thermal switching model with taking into account multiphonon tunneling ionization (MTI) of negative-U centers [28]. Experimental dependences are taken from [33].

According to [8,9] for various CGS compounds there is linear dependence between $\ln (\mathrm{j})$ and $\mathrm{F}$. See expression (2). One can see that electronic-thermal switching model with taking into account multiphonon tunneling ionization of negative-U centers very well explains such dependence for STAG (Fig. 2) and $\mathrm{Ge}_{2} \mathrm{Sb}_{2} \mathrm{Te}_{5}$ (225) (Fig. 3). In Fig. 2 this region is depicted by dotted line for $\mathrm{T}_{0}=250 \mathrm{~K}$.

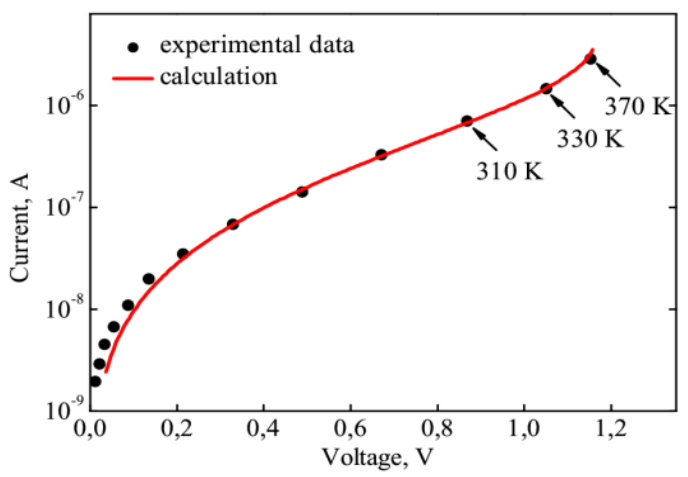

Figure 3. Subthreshold part of the CVC. The ambient temperature $\mathrm{T}_{0}=$ $300 \mathrm{~K}$. Calculated value of the film temperature is shown for several points [29]. Experimental CVC of a 40-nm thick 225 film from [34].

To demonstrate the role of heating CVC with (Fig. 4, 
dotted line) and without (Fig. 4, solid line) have been calculated [7].

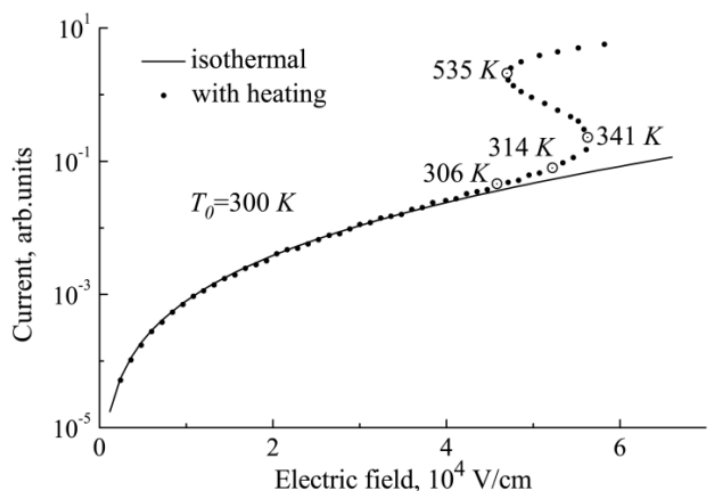

Figure 4. Current voltage characteristics calculated with (dotted line) and without (solid line) heating. $\mathrm{T}_{0}=300 \mathrm{~K}$ [7].

It may be seen that heating at the threshold point does not exceed 41 degrees, but one should not neglect it because the switching effect or S-shape in the electronic-thermal switching model occurs only for taking into account this heating. Secondly it is obvious the important role of the heating for existence of quasi linear region of $\mathrm{CVC}$ $(\ln j \sim E)$.

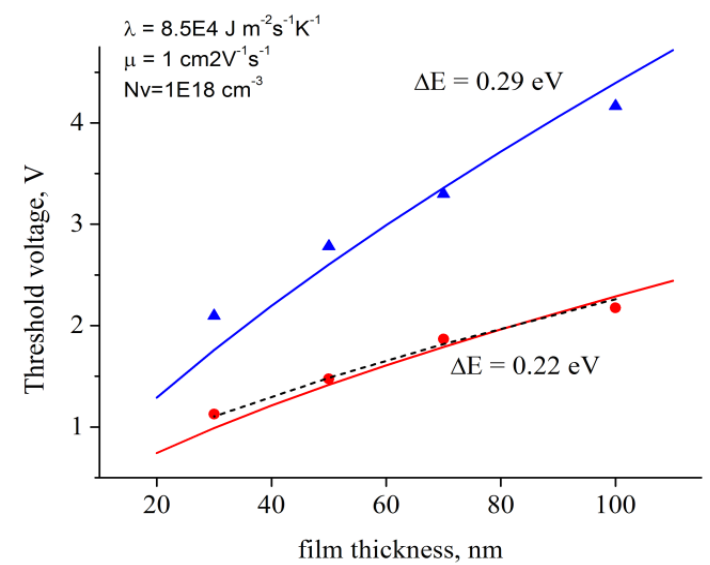

Figure 5. Dependence of $\mathrm{V}_{\text {th }}$ on $\mathrm{L}$ for $\mathrm{Ge}_{2} \mathrm{Sb}_{2} \mathrm{Te}_{5}$ films at room temperature 300 K. Data for as deposited films (triangles), for films amorphised by current pulse (circles) [20]. Solid lines for electronic-thermal switching model [29]. Dotted line for taking into account contact voltage $0,2 \mathrm{~B}$.

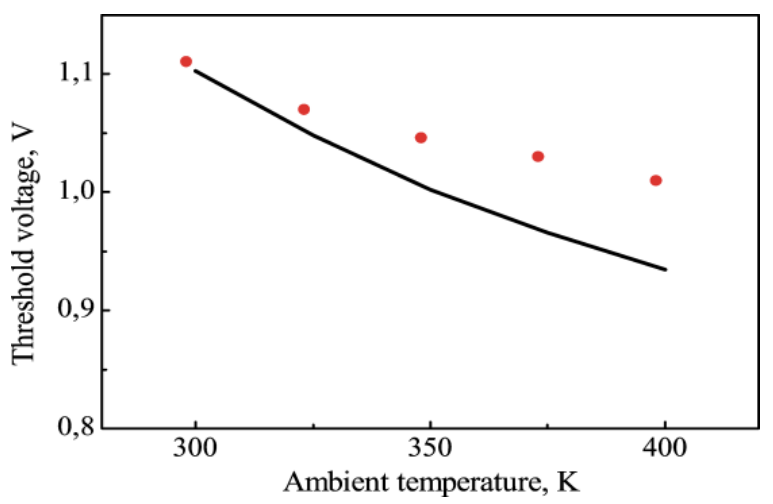

Figure 6. Dependence of $\mathrm{V}_{\text {th }}$ on $\mathrm{T}_{0}$ for $\mathrm{Ge}_{2} \mathrm{Sb}_{2} \mathrm{Te}_{5}$ films $\mathrm{L}=30 \mathrm{~nm}$ at room temperature $300 \mathrm{~K}[20]$. The solid line shows the calculated dependence. The same set parameters as for Fig. 5 has been used for calculation [29].

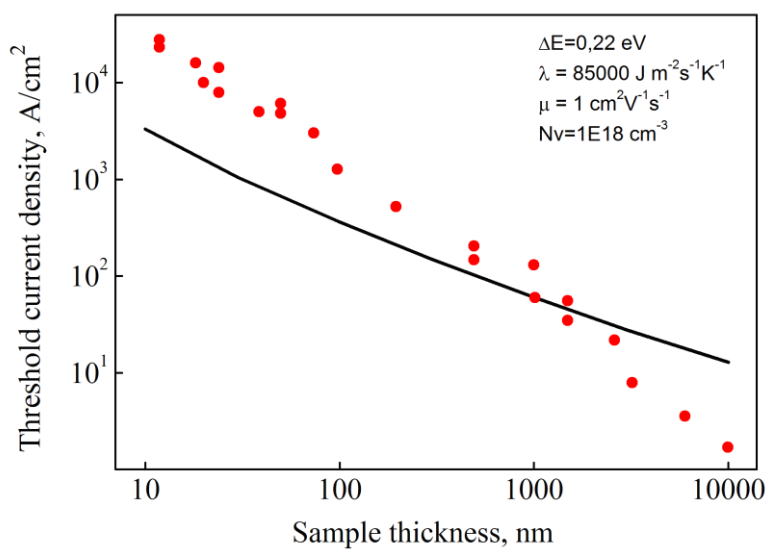

Figure 7. Threshold current density dependence on L. Experimental data are shown with symbols [35] and the solid line shows the calculated dependence [29].

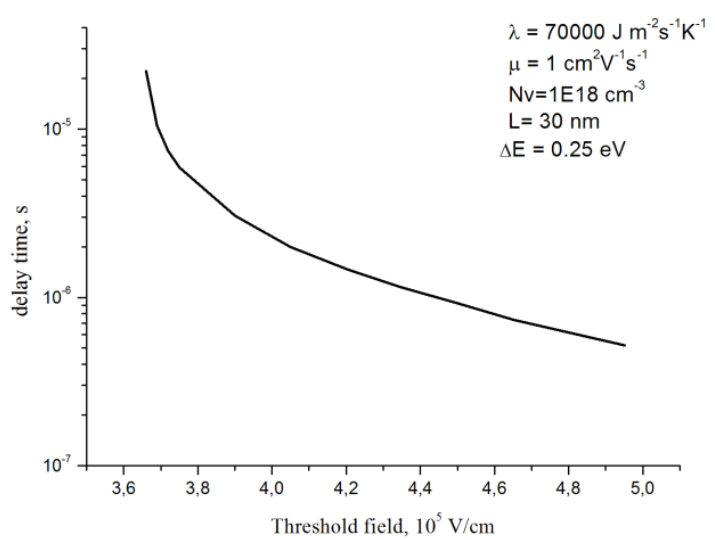

Figure 8. Delay time dependence on $\mathrm{V}$ calculated for $\mathrm{L}=50 \mathrm{~nm}$ [29].

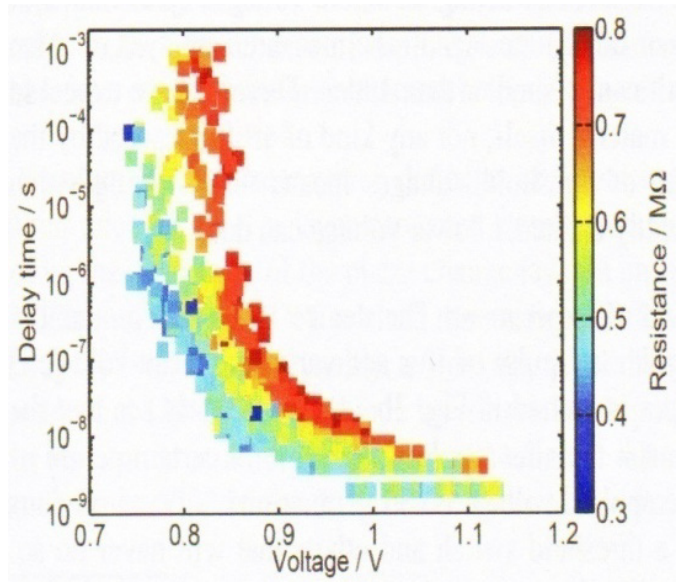

Figure 9. Delay time experimental dependence on V [36]. A lot of samples were used with different initial resistances (right column).

\section{Discussion}

Today for memory effect or recording, one has to use chalcogenide compound $\mathrm{Ge}_{2} \mathrm{Sb}_{2} \mathrm{Te}_{5}$ (GST or famous 225 compound). Resistance of crystalline phase of this compound many orders smaller then in amorphous phase. As for the memory effect, currently, very few researchers doubt 
that it is based on the "glass-crystal" phase transition. So memory cells based on the "glass-crystal" phase transition are known as Phase Change Memory (PCM) cells. Despite the fact that the physics of switching and memory effects has not yet been studied in sufficient detail, the phase memory technology has recently made significant progress. To demonstrate this, let us remind the main advances of recent years [7]. In December, 2006, at the IBM laboratory, a PCM cell prototype with an active region size of $3 \times 20 \mathrm{~nm}$ was fabricated. In [37] it became possible to decrease active region size to $20 \mathrm{~nm}$ also. In February, 2008, after 8 years of developments costing $\$ 2.5$ billion, the Intel Corporation in collaboration with the ST Microelectronics Company were the first to announce the batch production of PCM elements. The presented device with 128_Mbit memory was fabricated using 90_nm technology. In 2010, the Numonyx Company began to produce 1_Gbit memory elements. In 2011, Samsung Co. began to produce smartphones using PCM, which allowed a significant decrease in energy consumption and to increase the device's operational life.

Further progress in application very strong depends on understanding of microscopic mechanism of switching and memory effects. There are very comprehensive reviews on CGS properties [38-40], switching and memory effects [41-45], application and technology [46]. Adequate theory has to be able to explain all these data. The main aim of the present paper is to show how it fulfilled in the frame of the electronic-thermal model considering multiphonon tunneling ionization of negative- $U$ centers and heating.

\section{Conclusion}

The data presented in this paper show that the electronic-thermal model considering multiphonon tunneling ionization of negative- $U$ centers and heating accurately explains, in qualitative terms, all experimental features of the switching effect. The incomplete quantitative agreement can be caused by the model assumptions, e.g., disregarding the coordinate dependencies of temperature and electric field.

\section{Acknowledgements}

The author is grateful to Dr. N. A. Bogoslovskiy for substantial remarks. The research was supported by the Russian Academy of Science project N 24 and grant of Education and Science Ministry of Kazakhstan Republic 0938/GF2.

\section{REFERENCES}

[1] A.D. Pearson, Techn. Pap. VI Int. Congress of Glass, Washington, 357 (1962).
[2] B.T. Kolomiets. E.A.Lebedev. Radiotekh. Electron., 8, 2097 (1963).

[3] S.R. Ovshinsky, Phys. Rev. Lett., 21(20), 1450 (1968).

[4] K. D. Tsendin (ed.), Electronic Phenomena in Chalcogenide Glassy Semiconductors (in Russian) (Nauka, St. Petersburg,

[5] 1997), chap. 6 .

[6] B. T. Kolomiets, E. A. Lebedev, and I. A. Taksami, Sov.Phys. Semicond. 3, 267 (1969).

[7] B. T. Kolomiets, E. A. Lebedev, and I. A. Taksami, Sov.Phys. Semicond. 3, 621 (1969).

[8] N. A. Bogoslovskiy and K. D. Tsendin, Semiconductors, 46(5), 559-590 (2012).

[9] P. J. Walsh, R. Vogel, and E. J. Evans, Phys. Rev. 178, 1274 (1969).

[10] K. W. Boer and S. R. Ovshinsky, J. Appl. Phys. 41, 2675 (1970).

[11] N. F. Mott, Contemp. Phys. 10, 125 (1969).

[12] H. K. Henisch, Sci. Am. 221, 30 (1969).

[13] H. Fritzsche, J. Phys. Chem. Sol. 68, 878 (2007).

[14] V. B Sandomirskii and A. A. Sukhanov, Zarubezh. Elektron. 9, 68 (1976).

[15] A. G. Zabrodskii, S. M. Ryvkin, and I. S. Shlimak, JETP Lett. 18, 290 (1973).

[16] S. M. Ryvkin, JETP Lett. 15, 448 (1972).

[17] D. Adler, M. S. Shur, M. Silv

[18] D. Ielmini, Phys. Rev. B 78, 035308_1 (2008).

[19] D. Ielmini and Y. Zhang, J. Appl. Phys. 102, 054517 (2007).

[20] M. Nardone, V. G. Karpov, D. C. S. Jackson, and I. V. Karpov, Appl. Phys. Lett. 94, 103509 (2009).

[21] V. G. Karpov, Y. A. Kryukov, S. D. Savransky, and I. V. Karpov, Appl. Phys. Lett. 90, 123504 (2007).

[22] J. C. Male and A. C. Warren, Electron. Lett. 6, 567 (1970).

[23] B. T. Kolomiets, E. A. Lebedev, and K. D. Tsendin, Sov. Phys. Semicond. 5, 1369 (1971).

[24] S. D. Savransky, J. Ovonic Res. 1 (2), 25 (2005).

[25] K. D. Tsendin, J. Opt. Adv. Mater. 9, 3035 (2007).

[26] K. D. Tsendin, Phys. Status Solidi B 246, 1831 (2009).

[27] N. A. Bogoslowsky and K. D. Tsendin, Semiconductors 43, 1338 (2009).

[28] N. A. Bogoslovskiy and K. D. Tsendin, J. Opt. Adv. Mater. $11-12,1423$ (2011).

[29] N. A. Bogoslovskij and K. D. Tsendin, J. Non-Cryst. Sol. 357, 992 (2011).

[30] Bogoslovskiy N.A. // Phys. Status Solidi. B 250 (8), 1563 (2013) 
[31] D. L. Eaton, J. Am. Ceram. Soc. 47, 554 (1964).

[32] A. C. Warren, Electron. Lett. 5, 461 (1969).

[33] V. N. Abakumov, V. I. Perel', and I. N. Yassievich, Nonradiative Recombination in Semiconductors North_Holland, Amsterdam, 1991.

[34] P. I. Walsh, J. E. Hall, R. Nicolaides, S. Defeo, P. Callela, I. Kuchmas, and W. Doremus, J. Non_Cryst. Sol. 2, 107 (1970).

[35] D. Ielmini, Phys. Rev. B 78, 035308 (2008).

[36] S. A. Kostylev, Electron Device Lett. 30, 814 (2009).

[37] Salinga, M. The gradual nature of threshold switching / M. Salinga. M. Wimmer // E\PCOS 2012. Proceedings. Tampere, 2012. - Pp. 115-120.

[38] C. D. Wright, P. Shah, L. Wang, M. M. Aziz, A. Sebastian, and H. Pozidis, Appl. Phys. Lett. 97, 173104 (2010).

[39] M. Wuttig and N. Yamada, Nature Mater. 6, 824 (2007).
[40] W. Welnic and M. Wuttig, Mater. Today 11 (6), 20 (2008).

[41] D. Lencer, M. Salinga, and M. Wuttig, Adv. Mater. 23, 2030 (2011).

[42] A. L. Lacaita, Solid State Electron. 50, 24 (2006).

[43] A. Redaelli, A. Pirovano, A. Benvenuti, and A. L. Lacaita, J. Appl. Phys. 103, 111101 (2008).

[44] K. D. Tsendin and N. A. Bogoslovskiy, J. Opt. Adv. Mater. 11-12, 1429 (2011).

[45] S. Raoux, W. Welnic, and D. Ielmini, Chem. Rev. 110, 240 (2010).

[46] S. Sekander and C. D. Wright, J. Appl. Phys. 95, 504 (2004).

[47] G. W. Burr, M. J. Breitwisch, M. Franceschini, D. Garetto, K. Gopalakrishnan, B. Jackson, B. Kurdi, C. Lam, L. A. Lastras, A. Padilla, B. Rajendran, S. Raoux, and R. S. Shenoy, J. Vac. Sci. Technol. B 28, 223 (2010). 